\title{
CLINICAL RESEARCH ARTICLE Calcitriol trend following pediatric cardiac surgery and association with clinical outcome
}

\author{
Nina Acharya ${ }^{1,2}$, Dermot R. Doherty ${ }^{3}$, Nick Barrowman ${ }^{4}$, Gyaandeo Maharajh ${ }^{5}$, Tara Girolamo ${ }^{5}$, Katie O'Hearn $^{6}$ and J. Dayre McNally $^{1}$
}

\begin{abstract}
BACKGROUND: Consistent with accepted practice in stable ambulatory populations, the majority of ICU research has evaluated vitamin $D$ status using a single blood 25-hydroxyvitamin $D(25(\mathrm{OH}) \mathrm{D})$ level. Only a limited number of ICU studies have measured the active hormone, 1,25-dihydroxyvitamin D (calcitriol) and none have used change in calcitriol levels to evaluate axis functioning. The objective of this study was to describe the impact of Congenital Heart Disease (CHD) surgery on calcitriol levels and evaluate the relationship between change in postoperative levels and clinical course.

METHODS: Secondary analysis of a prospective cohort study of 56 children undergoing surgery for CHD.

RESULTS: Mean calcitriol levels dropped from $122.3 \pm 69.1 \mathrm{pmol} / \mathrm{L}$ preoperatively to $65.3 \pm 36.5 \mathrm{pmol} / \mathrm{L}(p<0.0001)$ at $\mathrm{PICU}$ admission. The majority $(61 \%, n=34)$ were unable to increase calcitriol levels in the $48 \mathrm{~h}$ immediately following surgery. Post operative trend in calcitriol was inversely related to cardiovascular dysfunction, fluid requirements, ventilatory support and PICU length of stay $(p<0.01)$.

CONCLUSION: CHD patients had significant dysfunction of the vitamin D axis immediately postoperatively, demonstrated by both a significant intraoperative decline in calcitriol and inability to increase levels. Interventional research will be required to determine whether the use of calcitriol, in addition to cholecalciferol, reduces postoperative illness severity.
\end{abstract}

Pediatric Research (2018) 84:254-260; https://doi.org/10.1038/s41390-018-0049-1

\section{INTRODUCTION}

Congenital heart disease (CHD) affects between 1 and $2 \%$ of all children, many of whom require surgery to prevent death, reduce morbidity, or improve quality of life. ${ }^{1}$ Benefits notwithstanding, children who undergo CHD surgery can endure a significant period of critical illness peri-operatively resulting in substantial suffering, prolonged periods of rehabilitation, and the potential to develop long-term morbidity. ${ }^{2-4}$ Improving clinical outcomes and reducing health care spending in this high-risk population will require enhanced understanding of both patient and operative factors that influence postoperative illness severity.

Recently, vitamin D status has been proposed as a potential effect modifier of clinical course and recovery following complex surgery and critical illness. ${ }^{5,6}$ In addition to the known essential role in calcium homeostasis and prevention of related diseases (i.e., rickets and hypocalcemic seizures), basic science and epidemiological research has also linked vitamin $D$ to the proper development and functioning of musculoskeletal, cardiac, and immune organ systems central to recovery from critical illness. ${ }^{7,8}$ Provided with substantial biological plausibility, the ICU research community has produced a large body of observational literature on this topic. ${ }^{6}$ Consistent with the accepted methodology, the vast majority have evaluated vitamin D status using a single blood measurement of the stable inactive precursor metabolite, 25hydroxyvitamin $\mathrm{D}$ or $25(\mathrm{OH}) \mathrm{D}$. Recent meta-analyses have estimated global vitamin $D$ deficiency (VDD) prevalence at ICU admission of $\sim 50 \%$ (using the $50 \mathrm{nmol} / \mathrm{L}$ threshold) and confirmed statistically significant associations between hormone level and clinical outcome including an approximate two-fold greater risk of mortality. ${ }^{5,9,10}$ Further, three small observational studies focused on CHD patients have also demonstrated high VDD rates postoperatively, ranging from 38 to $86 \%$, and associations with illness severity and increased requirement for ICU interventions. ${ }^{11-}$

${ }^{14}$ Although concerning, this has highlighted the potential value of vitamin D supplementation as a safe, straightforward, and inexpensive treatment to improve critical illness outcomes.

In addition to the recognized need for large confirmatory RCTs, a separate question that has emerged is whether a single measurement of blood $25(\mathrm{OH}) \mathrm{D}$ represents the ideal approach for assessing vitamin D status in the ICU. ${ }^{15}$ Although considered of limited diagnostic or prognostic value in most clinical and research settings, the active hormone, 1,25-dihydroxyvitamin $D$ $\left(1,25(\mathrm{OH})_{2} \mathrm{D}\right.$ or calcitriol), has been recognized to be of value in specific patient populations, including those with severe $25(\mathrm{OH}) \mathrm{D}$ deficiency, renal injury, and/or parathyroid dysfunction. ${ }^{16}$ Of the existing literature, only a few studies on adult patients following cardiac surgery, have shown an association between calcitriol concentrations and clinical outcome. ${ }^{17,18}$

No studies to date have evaluated whether change over time could be used as a measure of vitamin $D$ axis functioning. Although the short half-life $(12-24 \mathrm{~h})$ is often used as a rationale against calcitriol determination, this feature could be beneficial in dynamic illness states. The primary objective of this study was to investigate the impact of CHD surgery on functioning of the vitamin $\mathrm{D}$ axis, using serial postoperative calcitriol concentrations. The secondary objective was to determine the relationship

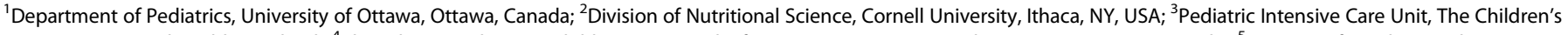

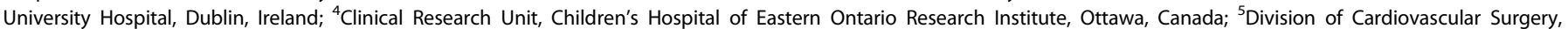
University of Ottawa, Ottawa, Canada and ${ }^{6}$ Children's Hospital of Eastern Ontario Research Institute, Ottawa, Canada

Correspondence: J Dayr McNally (dmcnally@cheo.on.ca) 
between illness severity and trend in postoperative calcitriol levels.

\section{METHODS}

Approval for the study was obtained from the Children's Hospital of Eastern Ontario's Institutional Review Board, and informed consent was obtained from all patients prior to enrollment.

\section{Subjects}

This study reports a secondary analysis of a prospective observational study evaluating the impact of anesthesia and CHD surgery on 25-hydroxyvitamin D levels. ${ }^{13}$ Briefly, eligible patients included children less than 18 years of age undergoing elective or semi-elective surgery for $\mathrm{CHD}$ requiring postoperative admission to the PICU. Patients previously enrolled in this study, another vitamin D study, requiring surgery for isolated pacemaker insertion or Blalock-Taussig shunts or missing two postoperative calcitriol measurements were excluded. Preoperative blood was collected from a control population of 25 children going to the operating room for minor procedures, as described in the original study. $^{13}$

\section{Measurements}

Five blood samples were collected during study enrollment. The first sample was collected preoperatively after anesthesia was induced and prior to surgical incision. Postoperative samples were collected at four different time points: PICU admission, $4-8 \mathrm{~h}$ after admission and morning of postoperative days 1 and 2. To further describe the nature of changes in calcitriol, four intraoperative samples were collected for the last 11 subjects at the following time points: immediately after cardiopulmonary bypass (CPB) initiation, on CPB prior to modified ultrafiltration (MUF), on CPB following MUF and $2 \mathrm{~mL}$ of the final MUF.

Blood $1,25(\mathrm{OH})_{2} \mathrm{D}$ concentrations were measured using validated immunoassays with ranges of $6-500 \mathrm{pmol} / \mathrm{L}$ and confirmed using 11 samples from the Vitamin D External Quality Assessment Scheme (DEQAS) with known concentrations of calcitriol. The experimental results matched the control values well with a correlation coefficient of 0.96 . The prevalence of low calcitriol was calculated using two different thresholds: (i) $40 \mathrm{pmol} / \mathrm{L}$, based on assay manufacturer suggestions; and (ii) $50 \mathrm{pmol} / \mathrm{L}$ in accordance with the literature review by Henderson et al. ${ }^{19}$ The $25(\mathrm{OH}) \mathrm{D}$ metabolite was measured as previously reported using a validated liquid chromatography-mass spectrometry procedure method. ${ }^{20}$

\section{Protocol}

Surgical and anesthetic care was determined by the cardiovascular surgeon, anesthesiologist and perfusionist. A balanced anesthesia technique (either fentanyl or remifentanil plus sevoflurane) was used. As per institution-accepted practice, infants under the age of 30 days were administered $30 \mathrm{mg} / \mathrm{kg}$ methylprednisone preoperatively. Moreover, CPB and cardioplegic arrest, including hypothermia as necessary, were performed as per institutional protocol and consistent with general standards of care. Every patient had an arterial and central venous catheter. Postoperatively, need for modified ultrafiltration (MUF) was determined by clinical state and residual circuit volume. Postoperative care was administered at the discretion of the pediatric intensivist with guidelines available for sedation, ventilation, and hypertension.

All data were prospectively recorded in a digital database. Patients' age, gender, weight, and ethnicity were recorded. A food frequency survey was administered to collect information on previous Vitamin D intake and preoperative nutritional status was determined using the Gomez and Waterlow approaches. ${ }^{21}$ Cardiac lesion diagnosis were collected along with the Risk-Adjusted Classification for Congenital Heart Surgery scores (RACHS), assigned according to Jenkins et al. ${ }^{22}$ CPB duration, prime circuit volume, CPB prime components, calcium administration, aortic cross clamp time, and total surgery time were collected during and immediately after surgery. During PICU stay, fluid administration, duration of mechanical ventilation, and length of stay were also collected. Creatinine levels greater than two standard deviations (SD) above normal values on postoperative day 1 were used to identify children with kidney dysfunction. ${ }^{23}$ The main clinical outcome of interest was cardiac dysfunction, which was defined as the need for catecholamine infusion postoperatively (dichotomous measure) and the inotrope score (continuous measure), calculated as described by Shore et al. ${ }^{24}$

\section{Data analysis}

Statistical analyses included population description, evaluation of calcitriol time course, and associations between calcitriol levels and clinical outcomes and predictors. Population characteristics were analyzed descriptively and presented as means and standard deviations for continuous variables (assuming the distribution did not fail normality testing) and percentages with counts and $95 \%$ confidence intervals for categorical variables.

The trends in calcitriol levels over time were evaluated using linear regressions and $t$-tests. Statistically significant changes in the prevalence of calcitriol deficiency was evaluated using the McNemar procedure. After testing for normality, a mixed linear model using a random intercept was employed to determine associations between predictors as well as clinical outcomes and calcitriol status over time. $p$-values $<0.05$ were considered significant. The SAS software (Version 9.4, Copyright SAS Institute Inc., Cary, NC) was used for analyses.

\section{RESULTS}

Study group demographics

The original study enrolled 58 children, of which 56 were included in this secondary analysis. Study participant baseline demographics and relevant clinical characteristics are shown in Table 1. Participants were $55 \%$ male, had a median age of 5.4 months (IQR $2.4,33.2)$, and $18 \%$ had a genetic disorder. Eighty-eight percent of participants were identified as Caucasian, followed by First Nations (7\%), African American (5\%), and other (5\%). Categorizing participant heart defects identified that $75 \%$ had RACHS 2 or RACHS 3 lesions. Only one study participant died, 5 months following surgery. A more detailed description, by RACHS category, is provided in Supplemental Table S1 (online).

Perioperative vitamin D measurements

Calcitriol. Compared to the control group $(n=25)$, there was no significant difference in mean preoperative calcitriol levels (135.7 \pm 50.6 vs. $122.3 \pm 69.1 \mathrm{pmol} / \mathrm{L}, p=0.4)$. Using the 40 and $50 \mathrm{pmol} /$ $\mathrm{L}$ thresholds for deficiency, only $3.6 \%(n=1)$ and $7.3 \%(n=2)$ of the CHD cohort met the definition for calcitriol deficiency preoperatively (Table 2), while none of the control cohort was below either threshold. Immediately following surgery, the group calcitriol levels were observed to decline to a mean of $65.3 \pm 36.5$ $\mathrm{pmol} / \mathrm{L}$, representing a highly significant change from the preoperative value $(p<0.0001)$ (Fig. 1). Due to the intraoperative decline, the number of participants meeting criteria for calcitriol deficiency at time of PICU admission increased significantly to $25.5 \%(n=14)$ and $36.4 \%(n=20)$ using the 40 and $50 \mathrm{pmol} / \mathrm{L}$ thresholds $(p=0.0005, p=0.0002)$. Furthermore, the prevalence of calcitriol deficiency continued to increase from PICU admission to postoperative day 2 with $44.4 \%$ of patients $(n=20)$ meeting the $40 \mathrm{pmol} / \mathrm{L}$ definition $(p=0.0076)$. Similarly, $48.9 \%$ of patients met the $50 \mathrm{pmol} / \mathrm{L}$ definition $(n=22)$, however this increase in deficiency from immediately postoperative was not significant. Postoperatively, only $39 \%(n=22)$ of patients had positive slopes for calcitriol level over time, with even fewer $(13 \%, n=7)$ demonstrating recovery to or above their preoperative levels. 
Calcidiol. The mean preoperative $250 \mathrm{HD}$ concentration was calculated as $58.6 \mathrm{nmol} / \mathrm{L}$ (SD 22.5), with $40 \%$ of patients experiencing deficiency ( $>50 \mathrm{nmol} / \mathrm{L}, n=22$ ) and $4 \%$ experiencing severe deficiency $(<25 \mathrm{nmol} / \mathrm{L}, n=2)$. At time of PICU admission, the mean 25OHD levels had fallen to $34.1 \mathrm{nmol} / \mathrm{L}$ (SD 14.6), with $85 \%(n=47)$ and $27 \%(n=15)$ having levels below 50 and 25 $\mathrm{nmol} / \mathrm{L}$, respectively. The average $250 \mathrm{HD}$ concentration increased by an average of only $2.7 \mathrm{nmol} / \mathrm{L}(95 \% \mathrm{Cl}:-0.33,5.7)$ over the first two postoperative days.

Table 1. Demographic and clinical characteristics of study participants

\begin{tabular}{ll}
\hline Patient characteristic & Study group $(n=56)$ \\
\hline Age, month; median (IQR) & $5.4(2.4,33.2)$ \\
Weight, kg; median (IQR) & $6.5(4.3,13.4)$ \\
Male sex; no. (\%) & $31(55 \%)$ \\
Season of surgery & \\
Summer; no. (\%) & $16(29 \%)$ \\
Fall; no. (\%) & $18(32 \%)$ \\
Winter; no. (\%) & $18(32 \%)$ \\
Spring; no. (\%) & $4(7 \%)$ \\
RACHS score & \\
Grade 1; no. (\%) & $9(16 \%)$ \\
Grade 2; no. (\%) & $23(41 \%)$ \\
Grade 3; no. (\%) & $19(34 \%)$ \\
Grade 4; no. (\%) & $5(9 \%)$ \\
Ethnicity & \\
Caucasian; no. (\%) & $49(88 \%)$ \\
$\quad$ Both parents; no. (\%) & $47(84 \%)$ \\
First nations; no. (\%) & $4(7 \%)$ \\
African American; no. (\%) & $3(5 \%)$ \\
Other $;$; no. (\%) & $3(5 \%)$ \\
Genetic syndrome & $1(2 \%)$ \\
Trisomy 21; no. (\%) & $1(2 \%)$ \\
Digeorge 22q11; no. (\%) & $1(2 \%)$ \\
Unknown; no. (\%) & \\
Premorbid axis disease & \\
Chronic kidney disease & \\
Chronic hepatic disease & \\
\hline IQR interquartile range, RACHS risk adjustment for congenital heart surgery \\
aPercentages may add up to more than $100 \%$ if more than one ethnicity \\
was recorded \\
bOther included Filipino (1) and South Asian (1) and Iranian (1) \\
\hline
\end{tabular}

Intraoperative calcitriol decline, timing, and mechanism Calcitriol dropped significantly $(p<0.0001)$ from preoperative measurement to the first intraoperative point, immediately following initiation of CPB (Fig. 2). No other significant intraoperative changes were observed. The median final level in the MUF was $6.8 \mathrm{pmol} / \mathrm{L}$ (IQR 3.5, 8.0).

Calcitriol and cardiac dysfunction

Of the study participants, $30.4 \%(n=17)$ required catecholamine infusions postoperatively. There was no significant difference in mean preoperative calcitriol levels between the groups that did and did not receive catecholamines $(118 \pm 66.5$ vs. $133 \pm 76.1$ $\mathrm{pmol} / \mathrm{L}, p=0.5)$. Postoperative calcitriol levels were compared between the two groups and are shown in Fig. 3. Although there was no difference at time of PICU admission, the group that received catecholamines had significantly lower calcitriol levels on postoperative day $1(57 \pm 28.7$ vs. $39 \pm 27.8 \mathrm{pmol} / \mathrm{L}, p=0.045)$ and postoperative day 2 (72 \pm 41.2 vs. $26 \pm 27.1 \mathrm{pmol} / \mathrm{L}, p=0.0004)$. A separate analysis confirmed this association, demonstrating that those who had a negative postoperative calcitriol slope were more likely to receive catecholamines, when compared to subjects with a positive calcitriol trend $(42 \%$ vs. $13 \%, p=0.02)$.

Calcitriol and clinical outcome measures

A mixed linear model was used to investigate the associations between both absolute calcitriol level and trend with established markers of organ dysfunction and morbidity (Table 3). Statistically significant associations were evident between the change in

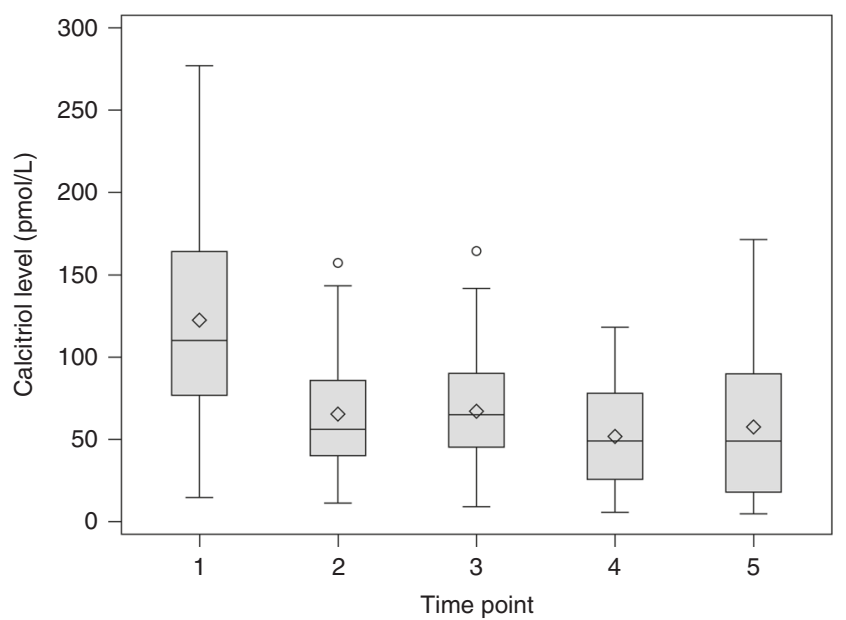

Fig. 1 Perioperative calcitriol levels. The box plot shows group mean (diamond) and median (horizontal line) calcitriol levels with IQRs. Time points: 1 , preoperative; 2 , pediatric intensive care unit (PICU) admission; 3, 4-8 h after PICU admission; 4, morning postoperative day $1 ; 5$, morning postoperative day 2

Table 2. Calcitriol status and deficiency rates by perioperative time point

\begin{tabular}{llll}
\hline Time point & $1,25(\mathrm{OH})_{2} \mathrm{D}_{\mathrm{NM}}$ & \% Deficiency $(50 \mathrm{pmol} / \mathrm{L})^{\mathrm{a}}$ & \% Deficiency $(40 \mathrm{pmol} / \mathrm{L})^{\mathrm{a}}$ \\
\hline Preoperative $(n=55)$ & $122.3(69.1)$ & $7.3(2-18)$ & $3.6(0-12)$ \\
PICU Admission $(n=55)$ & $65.3(36.5)$ & $36.4(23-50)$ & $25.5(15-39)$ \\
Postoperative $(n=46)$ & $67.0(35.4)$ & $30.4(18-46)$ & $17.4(8-31)$ \\
Postoperative, day $1(n=54)$ & $51.7(29.3)$ & $51.9(38-66)$ & $40.7(28-55)$ \\
Postoperative, day 2 $(n=45)$ & $57.9(42.9)$ & $48.9(34-64)$ & $44.4(30-60)$ \\
\hline
\end{tabular}

Values are means plus SDs or percentages with $95 \% \mathrm{Cls}$

$1,25(\mathrm{OH})_{2} \mathrm{D}$ 1,25-dihydroxyvitamin $\mathrm{D}$

a Deficiency defined as $25 \mathrm{OHD}$ level under 50 or $40 \mathrm{pmol} / \mathrm{L}$ 


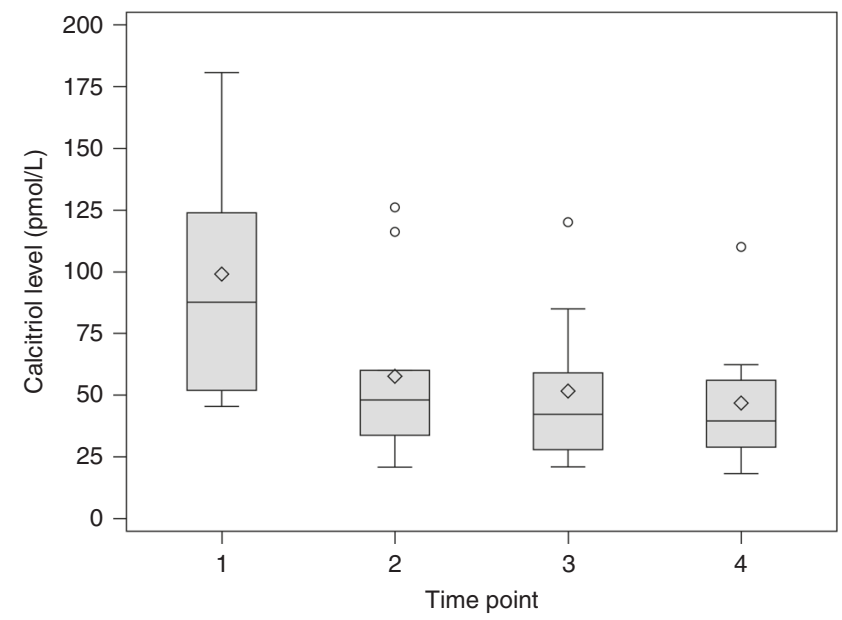

Fig. 2 Intraoperative calcitriol levels. The box plot shows group mean (diamond) and median (horizonal line) calcitriol levels with IQRs. Time points: 1 , preoperative; 2 , after CPB initiation; 3 , on CPB before modified ultrafiltration (MUF); 4, following MUF

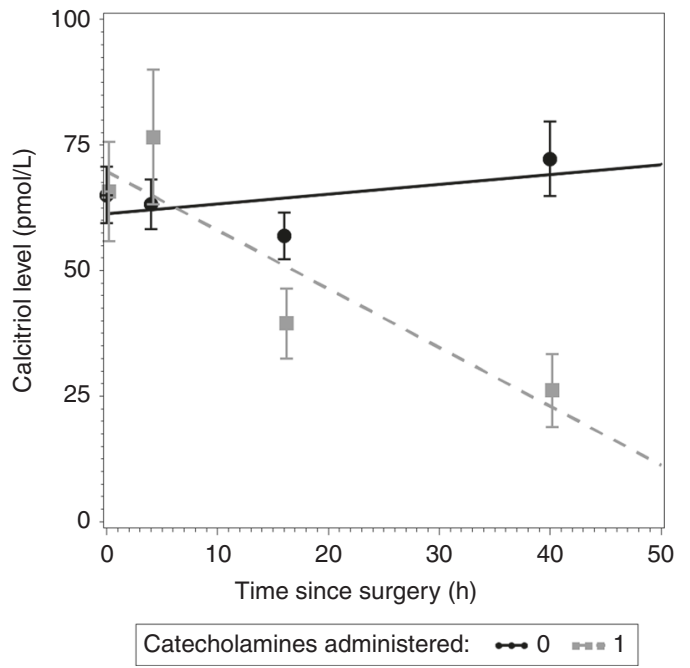

Fig. 3 Postoperative time course of calcitriol by receipt of catecholamine infusion. Graph shows mean calcitriol levels by catecholamine administration following surgery. Postoperative time points were estimated as $0,4,16$, and 40 hours from PICU admission

postoperative calcitriol levels and all clinical indicators of organ dysfunction and illness severity. For example, the postoperative calcitriol level was observed to be lower by $-26.6 \mathrm{pmol} / \mathrm{L}$ ( $\mathrm{SE} \pm 5.7$, $p=0.0001$ ) per day in the CHD patients requiring catecholamines postoperatively. All interactions remained significant after controlling for 25(OH)D status.

The relationship between calcium and calcitriol was also investigated. Of the 56 participants, 23 had at least one episode of hypocalcemia $(<1.1 \mathrm{mmol} / \mathrm{L})$ during first two postoperative days. As shown in Table 3, the mixed linear model did not demonstrate any difference in calcitriol levels (main effects or interaction), for the CHD patients experiencing hypocalcemia. Similarly, there was no significant relationship between calcitriol levels and the number of parental calcium doses administered (Table 3).

Predictors of postoperative calcitriol status

Table 4 presents the results of a mixed linear model used to investigate the associations between the trend in postoperative calcitriol level and preoperative, intraoperative, and immediate postoperative characteristics. When considering the predictors, only 1 of the 15 variables, the preoperative calcitriol level, was a significant predictor of postoperative level. When the interaction with time was considered, the preoperative calcitriol, ethnicity and renal function variables were also statistically associated with postoperative calcitriol levels. For example, having renal dysfunction (elevated creatinine on day 1) was associated with a 14.59 $\mathrm{pmol} / \mathrm{L}$ decrease in calcitriol per day compared to the group that did not exhibit signs of renal dysfunction.

\section{DISCUSSION}

Distinct from the majority of the critical care research to date, this study sought to evaluate functioning of the vitamin $D$ axis through serial measurements of the active hormone, calcitriol. Measurements were performed on blood collected immediately preoperatively, at time of PICU admission and at three additional time points over the first two postoperative days. Study results convincingly demonstrate the novel findings that the postoperative CHD patient is at significant risk for disruption of the vitamin $D$ axis and that a negative calcitriol trend is highly correlated with illness severity. Consistent with other ICU studies, individual calcitriol values immediately preoperatively or at time of PICU admission were not associated with clinical course.

Our longitudinal evaluation of calcitriol levels demonstrated that the majority of the CHD cohort experienced severe disruption and dysfunction of the vitamin D axis postoperatively. First, we observed a significant abrupt intraoperative decline in calcitriol levels, leading many children to have abnormally low blood levels at time of PICU admission. As a result, when applying either the 40 or $50 \mathrm{pmol} / \mathrm{L}$ threshold, $26 \%$ and $36 \%$ of the cohort, respectively, were deficient postoperatively compared to $3.6 \%$ and $7.3 \%$ preoperatively. The observed decline in calcitriol levels is consistent with findings from other studies reporting significant changes in both 25(OH)D and calcitriol levels following both adult and pediatric cardiac surgery. ${ }^{11,13,14,17,25}$ For example, we have previously reported a $40 \%$ intraoperative decline in $25(\mathrm{OH}) \mathrm{D}$ levels following CPB in children, and Krishnan et al. reported a 35\% decline in adults. ${ }^{25}$ The second central observation was that the average calcitriol levels either remained stable or declined further over the subsequent two postoperative days. Repeat evaluation of calcitriol status on the first and second postoperative day demonstrated that the proportion of the cohort with levels below $50 \mathrm{pmol} / \mathrm{L}$ had increased to $50 \%$, while only $13 \%$ of children were able to return to their preoperative levels. The observed trend suggests significant vitamin $\mathrm{D}$ axis dysfunction in some children following CHD surgery, as individuals with a functional vitamin $\mathrm{D}$ axis can maintain calcitriol concentrations by creating the active hormone from body stores of the precursor metabolite. For example, findings from studies on healthy and severely deficient children alike have demonstrated that children are capable of maintaining calcitriol levels above $50 \mathrm{pmol} / \mathrm{L}$ due to compensation by the parathyroid and renal organs. ${ }^{26,27}$ It is important to acknowledge that the kidneys and parathyroid glands of most VDD children have had considerable time (e.g., weeks or months) to compensate. In contrast, the children in our postoperative CHD cohort experienced a significant acute drop in $250 H D$, a very unnatural state for the vitamin D axis. Consequently, a transient decrease in calcitriol production would be expected, particularly given concomitant impairment of the parathyroid gland and kidneys secondary to surgery-induced ischemia and inflammation.

This study also sought to provide insight into the mechanisms contributing to the observed intraoperative and postoperative changes in calcitriol. First, similar to the previously observed significant drops in calcitriol immediately following CPB in adults and children, ${ }^{25,28}$ we documented that the majority of the intraoperative decline occurred after CPB. This change is most likely 
Table 3. Mixed linear model fits for postoperative calcitriol level

\begin{tabular}{|c|c|c|c|c|c|c|}
\hline \multirow[b]{2}{*}{ Outcome variable } & \multicolumn{3}{|c|}{$\begin{array}{l}\text { Main effect of outcome (difference at baseline } \\
\text { per outcome unit) }\end{array}$} & \multicolumn{3}{|c|}{$\begin{array}{l}\text { Interaction with time }{ }^{a} \text { (difference in slope per } \\
\text { outcome unit) }\end{array}$} \\
\hline & Effect estimate & SE & $p$-Value & Effect estimate $^{b}$ & SE & $p$-Value \\
\hline Inotrope score & -0.17 & 0.70 & 0.81 & -1.52 & 0.42 & 0.0004 \\
\hline PICU length of stay (days) & 0.28 & 0.63 & 0.66 & -1.46 & 0.45 & 0.0015 \\
\hline Total fluid intake $(\mathrm{mL} / 10 \mathrm{~kg})^{\mathrm{c}}$ & 0.03 & 0.03 & 0.37 & -0.69 & 0.18 & 0.0001 \\
\hline Total No of fluid boluses ${ }^{c}$ & -0.88 & 0.67 & 0.19 & -1.39 & 0.45 & 0.0022 \\
\hline Postoperative hypocalcemia & -9.6 & 8.8 & 0.28 & -0.74 & 5.63 & 0.90 \\
\hline Total no. of calcium boluses & 1.27 & 1.82 & 0.49 & -1.25 & 1.14 & 0.27 \\
\hline
\end{tabular}

explained through hemodilution, although absorption by tubing or membranes represents another possibility. While CPB accounted for the majority of the intraoperative fall, evaluation of group average calcitriol levels shows an ongoing decline over the course of the operation. This is also not unexpected as intraoperative changes in $25(\mathrm{OH}) \mathrm{D}$ have been reported in non-CPB surgical populations, with inflammation (capillary leak), blood loss, and fluid administration provided as the most likely explanations. ${ }^{29}$ Finally, given the length of some surgeries, the decline in calcitriol may also relate to a mismatch between calcitriol consumption and production. We performed mixed linear regression modeling and identified three variables associated with a negative postoperative trend; elevated day 1 creatinine, preoperative calcitriol concentration, and nonCaucasian ethnicity. The documented relationship between kidney function and calcitriol levels is expected, and has been elegantly demonstrated previously by Zitterman and colleagues in the adult cardiac surgical setting. ${ }^{18}$ Although no relationship was evident between preoperative $25(\mathrm{OH}) \mathrm{D}$, we did observe that higher preoperative calcitriol levels were highly associated with an increasingly negative postoperative calcitriol trend. Although counterintuitive, this observation does align with studies showing that calcitriol levels are often elevated in the setting of 25(OH)D deficiency, due to overcompensation by functional parathyroid and renal glands. ${ }^{30}$ Although neither the pre- or postoperative $25(\mathrm{OH}) \mathrm{D}$ levels were associated with the calcitriol trend, it is important to note that very few CHD patients had $25(\mathrm{OH}) \mathrm{D}$ above target levels (75 $\mathrm{nmol} / \mathrm{L}$ ). Consequently it may be possible to optimize function of the vitamin $D$ axis, maintaining more normal perioperative calcitriol levels, through cholecalciferol supplementation.

In addition to assessing the mechanism of the vitamin $D$ axis dysfunction, we also sought to evaluate and quantify relationships between calcitriol and postoperative illness severity. Our first observation was that there was no relationship between either the calcitriol concentrations preoperatively or at time of PICU admission and postoperative cardiovascular dysfunction (e.g., catecholamine infusion requirement) or any other measure of illness severity. This finding contrasts with previous observations by ourselves and others for the $25(\mathrm{OH}) \mathrm{D}$ metabolite. ${ }^{12,13}$ However, it is consistent with pediatric ICU studies failing to demonstrate that admission calcitriol concentrations were independently associated with clinical course. $^{31-33}$ For example, a study of 337 critically ill Canadian children reported that admission calcitriol was not associated with measures of clinical outcome including catecholamine administration and PICU length of stay. ${ }^{32}$ Separate from our investigations of the absolute pre- and postoperative calcitriol levels, we provide the novel observation of a highly significant association between a negative trend in calcitriol levels and greater postoperative illness severity. For example, the group of children who required catecholamines postoperatively had a decline in calcitriol levels by $-27 \mathrm{pmol} / \mathrm{L}$ per day, when compared to those who did not. Despite the relatively small sample size, very strong statistical association were observed between postoperative calcitriol trend and all outcome measures including inotrope score, PICU length of stay, and fluid requirements. Although few pediatric surgical studies exist for comparison, a few adult cardiac surgical studies have identified low calcitriol to be associated with increased risk of worse clinical outcome. ${ }^{17,18,34}$

A causal relationship between disruption of the vitamin $D$ axis and worse clinical outcome, particularly the cardiovascular and immune systems, does have biological plausibility. The negative impact of low calcitriol levels could be mediated indirectly through electrolyte disturbance, particularly hypocalcemia. While vitamin D status has been related to blood calcium levels in other ICU studies, ${ }^{35}$ the lack of an association in this study would suggest that the observed negative effects of vitamin $D$ axis dysfunction and calcitriol deficiency were not mediated via hypocalcemia. Alternatively, vitamin D can exert more direct effects on organ function. Consider, for example, that vitamin D receptors (VDR) have been identified on cardiomyocytes, dendritic cells, T cells, and macrophages allowing them to mediate cardiac and immune function. Cell-based and animal model studies have demonstrated that acute changes in calcitriol concentrations can rapidly influence cardiac cell functioning. ${ }^{36}$ Similarly, $T$ cells that have been chronically activated may be turned off by the docking of calcitriol to dendritic and T cells. ${ }^{37}$ Therefore, a rapid and sustained decline in calcitriol concentrations during critical illness may promote cytokines release and inflammation through activation of Type 1T helper cells. Despite the biological plausibility, given the observational study design, it is not possible to strongly endorse a causal relationship between disruption of the vitamin $D$ axis and worse clinical outcome following pediatric cardiac surgery.

This is the first pediatric study to use the postoperative trend in calcitriol to investigate the functioning of the vitamin $D$ axis and explore its relationship with clinical outcome. Although this study has multiple strengths, a number of limitations should be acknowledged. First, the study had a small sample size $(n=56)$ limiting mixed linear modeling and investigation of characteristics associated with calcitriol trend. For example, the relationship between non-Caucasian ethnicity and calcitriol trend could 
Table 4. Mixed linear model fits for postoperative calcitriol level predictors

\begin{tabular}{|c|c|c|c|c|c|c|}
\hline \multirow[b]{2}{*}{ Predictor variable } & \multicolumn{3}{|c|}{$\begin{array}{l}\text { Main effect of predictor (difference at baseline } \\
\text { per predictor unit) }\end{array}$} & \multicolumn{3}{|c|}{$\begin{array}{l}\text { Interaction with time }{ }^{\mathrm{a}} \text { (difference in slope per } \\
\text { predictor unit) }\end{array}$} \\
\hline & Effect estimate & SE & $p$-Value & Effect estimate $^{a}$ & $\mathrm{SE}$ & $p$-Value \\
\hline Age (months) & -0.02 & 0.10 & 0.80 & 0.09 & 0.07 & 0.21 \\
\hline Preoperative weight $(\mathrm{kg})$ & -0.16 & 0.37 & 0.67 & 0.25 & 0.24 & 0.30 \\
\hline RACHS score 3 and $4^{b}$ & 16.74 & 8.71 & 0.06 & -8.85 & 5.62 & 0.12 \\
\hline Summer & -3.90 & 17.65 & 0.83 & -4.10 & 11.07 & 0.71 \\
\hline Fall & -24.19 & 17.39 & 0.17 & -8.58 & 10.78 & 0.43 \\
\hline Winter & -19.53 & 17.42 & 0.27 & -9.86 & 10.86 & 0.37 \\
\hline Non-Caucasian ethnicity ${ }^{d}$ & -8.42 & 11.68 & 0.47 & -19.86 & 7.46 & 0.009 \\
\hline Preoperative $1,25(\mathrm{OH})_{2} \mathrm{D}(\mathrm{pmol} / \mathrm{L})$ & 0.37 & 0.05 & $<0.0001$ & -0.23 & 0.04 & $<0.0001$ \\
\hline $\mathrm{CPB}(\mathrm{Y} / \mathrm{N})$ & -33.03 & 16.57 & 0.051 & 5.96 & 10.03 & 0.55 \\
\hline CPB time (min) & $-9.74 \times 10^{-3}$ & 0.06 & 0.86 & -0.01 & 0.03 & 0.75 \\
\hline Total surgery duration (min) & $-6.30 \times 10^{-4}$ & 0.04 & 0.99 & $2.99 \times 10^{-3}$ & 0.02 & 0.89 \\
\hline Cross clamp time (min) & 0.03 & 0.08 & 0.67 & -0.05 & 0.05 & 0.36 \\
\hline Prime volume $(\mathrm{mL} / \mathrm{kg})$ & -0.08 & 0.33 & 0.829 & 0.02 & 0.20 & 0.94 \\
\hline \multicolumn{7}{|l|}{ Renal dysfunction } \\
\hline Elevated day 1 creatinine & -8.68 & 11.15 & 0.4398 & -14.59 & 7.10 & 0.04 \\
\hline \multicolumn{7}{|c|}{$\begin{array}{l}\text { CPB cardiopulmonary bypass } \\
\text { a Units for the effect estimate are pmol/L/day } \\
{ }^{b} \text { Against reference group of RACHS scores } 1 \text { and } 2 \\
{ }^{c} \text { Season indicates season at time of surgery-spring is the reference group } \\
\text { d Ethnicity reference category represented patients with at least one parent identified as Caucasian }_{\text {e }} \text { e Model was run using data from PICU admission to postoperative day } 2\end{array}$} \\
\hline
\end{tabular}

perhaps be explained by low sample size $(n=9)$ or by the low preoperative levels in this population. Furthermore, these results are based on findings from a single Canadian centre, thus limiting generalizability of results. Difference in vitamin D supplementation approaches, cardiac lesions repaired, and approaches to cardiopulmonary bypass at our centre could lead to differences in the severity of post-operative disruption of the vitamin $D$ axis. Additional observational studies and interventional trials involving administration of the active hormone will be needed to confirm our findings.

In conclusion, this secondary analysis clearly demonstrates the negative effect of CHD surgery on the vitamin D axis. Importantly, this study also documented that a more negative trend in calcitriol levels had a very strong association with postoperative illness severity. Interventional studies will be required to determine whether optimization of vitamin D status through supplementation leads to improved clinical outcome or simply adds cost and toxicity risk. Consider, for example, the scenario where the decline in calcitriol levels is an adaptive response by the body to acute stress, similar to sick euthyroid syndrome, and replacement of a potentially toxic hormone is counterproductive. That said, results from our mechanistic work suggest the majority of the calcitriol decline is not intentional, instead occurring secondary to $C P B$, the associated fall in 25OHD substrate, and postoperative kidney dysfunction. Initial clinical trials should consider cholecalciferol regimens alone as multiple clinical trials have demonstrated two- to three-fold elevations of $25(\mathrm{OH}) \mathrm{D}$ and $1,25(\mathrm{OH}) 2 \mathrm{D}$ in response to large enteral doses. ${ }^{38}$ However, enteral cholecalciferol supplementation may not be sufficient in many critically ill children due to malabsorption and dysfunction of organ central to the vitamin D axis (kidney, liver, parathyroid gland). ${ }^{39,40}$ The pediatric CHD field benefits from a recent pilot trial by Leaf and colleagues demonstrating not only that it is safe to administer intravenous calcitriol during critical illness, but that it can rapidly induce biochemical changes. ${ }^{33}$

\section{FUNDING}

The study was supported through grants from the Children's Hospital of Eastern Ontario Research Institute (Ottawa, Ontario, Canada) and Department of Anesthesia, University of Ottawa (Ottawa, Ontario, Canada). Research fellowship funding to JDM was provided by the Children's Hospital of Eastern Ontario Popham Foundation (Ottawa, Ontario, Canada). NA was supported through a summer studentship from the Children's Hospital of Eastern Ontario Research Institute (Ottawa, Ontario, Canada).

\section{ADDITIONAL INFORMATION}

The online version of this article (https://doi.org/10.1038/s41390-018-0049-1) contains supplementary material, which is available to authorized users.

Competing interests: The authors declare no competing interests.

Publisher's note: Springer Nature remains neutral with regard to jurisdictional claims in published maps and institutional affiliations. 


\section{REFERENCES}

1. Van Der Linde, D. et al. Birth prevalence of congenital heart disease worldwide: a systematic review and meta-analysis. J. Am. Coll. Cardiol. 58, 2241-2247 (2011).

2. Brix-Christensen, V. The systemic inflammatory response after cardiac surgery with cardiopulmonary bypass in children. Acta Anaesthesiol. Scand. 45, 671-679 (2001).

3. Gazit, A. Z., Huddleston, C. B., Checchia, P. A., Fehr, J. \& Pezzella, A. T. Care of the pediatric cardiac surgery patient--part 1. Curr. Probl. Surg. 47, 185-250 (2010).

4. McEwan, A. Aspects of bleeding after cardiac surgery in children. Paediatr. Anaesth. 17, 1126-1133 (2007)

5. McNally, J. D. et al. Vitamin D deficiency in critically ill children: a systematic review and meta-analysis. Crit. Care 21, 287 (2017).

6. Iglar, P. J. \& Hogan, K. J. Vitamin D status and surgical outcomes: a systematic review. Patient Saf. Surg. 9, 14 (2015)

7. Amrein, K. \& Venkatesh, B. Vitamin D and the critically ill patient. Curr. Opin. Clin. Nutr. Metab. Care 15, 188-193 (2012).

8. Lee, P. Vitamin D metabolism and deficiency in critical illness. Best. Pract. Res Clin. Endocrinol. Metab. 25, 769-781 (2011).

9. de Haan, K., Groeneveld, A. B. J., de Geus, H. R. H., Egal, M. \& Struijs, A. Vitamin D deficiency as a risk factor for infection, sepsis and mortality in the critically ill: systematic review and meta-analysis. Crit. Care 18, 660 (2014).

10. Zhang, Y.-P., Wan, Y.-D., Sun, T.-W., Kan, Q.-C. \& Wang, L.-X. Association between vitamin $D$ deficiency and mortality in critically ill adult patients: a meta-analysis of cohort studies. Crit. Care 18, 684 (2014).

11. Turan, A. et al. Low vitamin D concentration is not associated with increased mortality and morbidity after cardiac surgery. PLoS ONE 8, e63831 (2013).

12. Zittermann, A. et al. Vitamin D status and the risk of major adverse cardiac and cerebrovascular events in cardiac surgery. Eur. Heart J. 34, 1358-1364 (2013).

13. McNally, J. D. et al. Impact of anesthesia and surgery for congenital heart disease on the vitamin d status of infants and children: a prospective longitudinal study. Anesthesiology 119, 71-80 (2013).

14. Graham, E. M. et al. Vitamin D status in neonates undergoing cardiac operations: relationship to cardiopulmonary bypass and association with outcomes. J. Pediatr. 162, 823-826 (2013).

15. Krishnan, A. \& Venkatesh, B. Vitamin D measurement in the intensive care unit: methodology, clinical relevance and interpretation of a random value. Inflamm. Allergy Drug Targets 12, 230-238 (2013).

16. Cianferotti, L. et al. The clinical use of vitamin D metabolites and their potential developments: a position statement from the European Society for Clinical and Economic Aspects of Osteoporosis and Osteoarthritis (ESCEO) and the International Osteoporosis Foundation (IOF). Endocrine 50, 12-26 (2015).

17. Zittermann, A. et al. Circulating 25-hydroxyvitamin D and 1,25-Dihydroxyvitamin $D$ concentrations and postoperative infections in cardiac surgical patients: The CALCITOP-study. PLOS ONE 11, 1-12 (2016).

18. Zittermann, A. et al. 25-hydroxyvitamin D, 1,25-dihydroxyvitamin D and postoperative outcome in cardiac surgery. J. Clin. Endocrinol. Metab. 100, 72-80 (2015).

19. Henderson, R. C. Vitamin D Levels in children with cystic fibrosis. South Med. J. 90, 378 (1997).

20. Maunsell, Z., Wright, D. J. \& Rainbow, S. J. Routine isotope-dilution liquid chromatography-tandem mass spectrometry assay for simultaneous measurement of the 25-hydroxy metabolites of vitamins D2 and D3. Clin. Chem. 51, 1683-1690 (2005)
21. Grover, Z. \& Ee, L. C. Protein energy malnutrition. Pediatr. Clin. North Am. 56, 1055 (2009).

22. Jenkins, K. J. et al. Consensus-based method for risk adjustment for surgery for congenital heart disease. J. Thorac. Cardiovasc Surg. 123, 110-118 (2002).

23. Schwartz, G. J., Haycock, G. B. \& Spitzer, A. Plasma creatinine and urea concentration in children: normal values for age and sex. J. Pediatr. 88, 828-830 (1976).

24. Shore, S. et al. Usefulness of corticosteroid therapy in decreasing epinephrine requirements in critically ill infants with congenital heart disease. Am. J. Cardiol. 88, 591-594 (2001).

25. Krishnan, A. et al. Acute fluid shifts influence the assessment of serum vitamin D status in critically ill patients. Crit. Care 14, R216 (2010).

26. Elzouki, A. Y., Markestad, T., Elgarrah, M., Elhoni, N. \& Aksnes, L. Serum concentrations of vitamin D metabolites in rachitic Libyan children. J. Pediatr. Gastroenterol. Nutr. 9, 507-512 (1989).

27. Christensen, M. H. E., Lien, E. A., Hustad, S. \& Almas, B. Seasonal and age-related differences in serum 25-hydroxyvitamin $D, 1,25$-dihydroxyvitamin $D$ and parathyroid hormone in patients from Western Norway. Scand. J. Clin. Lab Invest 70, 281-286 (2010).

28. Abou Zahr R, Faustino EVS, Carpenter T, Kirshbom P, Hall EK, Fahey JT, Kandil SB. J. Intensive Care Med. 32, 508-513 (2017). https://doi.org/10.1177/ 0885066616652077

29. Reid, D. et al. The relation between acute changes in the systemic inflammatory response and plasma 25-hydroxyvitamin D concentrations after elective knee arthroplasty. Am. J. Clin. Nutr. 93, 1006-1011 (2011).

30. Mangin, M., Sinha, R. \& Fincher, K. Inflammation and vitamin D: the infection connection. Inflamm. Res 63, 803-819 (2014).

31. Nair, P. et al. Significant perturbation of vitamin D-parathyroid-calcium axis and adverse clinical outcomes in critically ill patients. Intensive Care Med. 39, 267-274 (2013).

32. McNally, J. D., Menon, K., Lawson, M. L., Williams, K. \& Doherty, D. R. 1,25-Dihydroxyvitamin $D$ levels in pediatric intensive care units: risk factors and association with clinical course. J. Clin. Endocrinol. Metab. 100, 2942-2945 (2015).

33. Leaf, D. E., Raed, A., Donnino, M. W., Ginde, A. A. \& Waikar, S. S. Randomized controlled trial of calcitriol in severe sepsis. Am. J. Respir. Crit. Care Med. 190, 533-541 (2014).

34. Börgermann, J. et al. 1,25-Dihydroxyvitamin D fluctuations in cardiac surgery are related to age and clinical outcome*. Crit. Care Med. 40, 2073-2081 (2012).

35. $\mathrm{Hu}, \mathrm{J}$. et al. Changes in the calcium-parathyroid hormone-vitamin $\mathrm{d}$ axis and prognosis for critically ill patients: a prospective observational study. PLOS ONE 8 , e75441 (2013).

36. Green, J. J., Robinson, D. A., Wilson, G. E., Simpson, R. U. \& Westfall, M. V. Calcitriol modulation of cardiac contractile performance via protein kinase C. J Mol. Cell Cardiol. 41, 350-359 (2006).

37. Cantorna, M. T. \& Waddell, A. The vitamin D receptor turns off chronically activated T cells. Ann. N. Y. Acad. Sci. 1317, 70-75 (2014)

38. Amrein, K. et al. Effect of high-dose vitamin D3 on hospital length of stay in critically ill patients with vitamin D deficiency: the VITdAL-ICU randomized clinical trial. JAMA 312, 1520-1530 (2014).

39. Greenbaum, L. A. et al. Intravenous calcitriol for treatment of hyperparathyroidism in children on hemodialysis. Pediatr. Nephrol. 20, 622-630 (2005).

40. Seeherunvong, W. et al. Paricalcitol versus calcitriol treatment for hyperparathyroidism in pediatric hemodialysis patients. Pediatr. Nephrol. 21, 1434-1439 (2006). 